\title{
Changing Beliefs and Memories Through Dream Interpretation
}

\author{
GIULIANA A. L. MAZZONI, ${ }^{1 *}$ ELIZABETH F. LOFTUS, ${ }^{2}$ \\ AARON SEITZ ${ }^{2}$ and STEVEN J. LYNN ${ }^{3}$ \\ ${ }^{1}$ University of Florence, Italy \\ ${ }^{2}$ University of Washington, USA \\ ${ }^{3}$ State University of New York, Binghamton, USA
}

\begin{abstract}
SUMMARY
Autobiographical memory is malleable, but how much can we change people's beliefs and memories about the past? We approached this question with a method designed to supply subjects with a highly personalized suggestion about what probably happened in their childhood. In the current study, one group of subjects (the 'Dream' subjects) had their dreams interpreted to indicate that they had experienced a critical childhood event (e.g. being harassed by a bully) before the age of 3 . Relative to control subjects who did not receive personalized suggestion, the Dream subjects were more likely to increase their belief that they had the critical experience, and approximately half of these also produced concrete memory reports. These findings are discussed in terms of their implications for autobiographical memory, and also for psychotherapy practice. Copyright (C) 1999 John Wiley \& Sons, Ltd.
\end{abstract}

We know that autobiographical memory is malleable, but how much can be change people's beliefs and memories about the past? Many researchers have explored this question, and have devised a variety of paradigms for doing so. For example, one paradigm involves family members who collaborate with the researchers in providing false suggestions about the childhood of their relative (Loftus and Pickrell, 1995; Hyman et al., 1995). Another paradigm involves using misinformation and suggestion to get people to remember highly improbable memories of infancy (Malinoski and Lynn, 1996).

To explore this question we have devised a novel method of modifying people's beliefs about the past with personalized suggestion. Our method attempts to simulate more closely the kinds of activities that go on in some psychotherapy settings (Poole et al., 1995). In our first study (Mazzoni et al., 1997) subjects reported on their childhood experiences on two occasions, separated by 3-4 weeks. Between these sessions, some subjects were exposed to a brief therapy simulation in which an expert clinician analysed a dream report that they had brought to the session. No matter 
what the content of their dream, the subject received the suggestion that their dream was indicative of having experienced, before age 3 , certain events such as having been lost in a public place or abandoned by their parents. Although subjects had previously indicated that they had not had these critical experiences before age 3, this 30-minute therapy simulation led many subjects to develop new beliefs about their past. Relative to controls who had not received the personalized suggestion, these 'therapy' subjects were far more likely to develop a false belief that before age 3 they had been lost in a public place, that they had felt lonely and lost in an unfamiliar place, and that they had been abandoned by their parents.

In a follow-up study using a similar procedure we showed that subjects who had their dreams interpreted to indicate that they had experienced a very dangerous event before age 3 would later report an increased belief that the danger event had occurred to them. The new beliefs that were generated by the dream interpretation were maintained for at least four weeks. Taken together these studies show the powerful effects on beliefs that this simple 30-minute manipulation could have.

When the beliefs of our subjects changed as a result of this experience, as they did in all the studies that we have conducted, what exactly is it that changed? The subjects in the studies mentioned above (e.g. Mazzoni et al., 1997) had been given a Life Events Inventory which asked how confident they were that they had had certain critical experiences, such as being rescued from danger before age 3 . They responded using an 8 -point scale, ranging from $1=$ definitely didn't happen to $8=$ definitely did happen. The main dependent variable of interest was whether they increased their confidence that the event happened, and if so how much, and whether the intervening session was responsible for the change. Not only were subjects highly inclined to increase their confidence after the dream session, as indicated by the proportion who increased, but the degree of upward shift was also strongly influenced. Do these data indicate that subjects remember some actual experience that fits the critical category, that is, an actual memory of being lost of being in danger? Or have we simply influenced the belief that they had the experience, without creating in their minds any particular memory? We cannot answer that question since we never inquired about whether subjects had a concrete memory to tell us about. One of the main purposes of the current study was to provide this kind of inquiry so as to determine more precisely what the consequences of our manipulation might be.

Another goal of the current study was to extend our prior results to a novel critical item, that of being harassed by a bully before age 3. Most of the subjects in the current research had their dreams interpreted to suggest that they had probably had this experience when they were young. We chose the 'bully' item since it would have been at least mildly traumatic if it had happened, and might even be more than mildly traumatic if it involved physical harm. Could such 'traumatic' experiences be suggested in this way?

So, in the current research, after subjects had gone through a dream session intended to suggest that they had been bullied before age 3 , if they told us that their belief had increased, that is, they demonstrated that they were now more confident that they had been bullied in childhood, we probed them for more information. We probed by explicitly asking them to remember as much as they could about a few possible experiences from their childhood. That list included the critical item 'harassed by a bully'. We recorded whether the subjects produced a concrete memory, and collected their verbatim responses for later analysis. 
Finally, a third issue motivating the current research was the question of who is particularly susceptible to this type of manipulation of the autobiography? We hypothezed that two individual difference variables might matter. The first was the Dissociative Experiences Scale (DES) which taps into the extent to which a person has lapses of memory and attention or fails to integrate awareness, thought and memory (Bernstein and Putnam, 1986). This scale has already been extensively used in studies on individual differences in suggestibility and has been found to correlate with suggestibility, in the direction of greater suggestibility for those with more memory lapses (e.g. Hyman and Billings, in press). The second variable was an instrument designed to measure subjects' a priori beliefs about dreams. For example, it asked whether they believed that dreams reveal meaning or whether dreams enable one to discover something about the self. We predicted that those who had a propensity to believe in dream interpretation would be more influenced by our manipulation.

\section{METHOD}

\section{Subjects}

An initial pool of 159 undergraduates from the University of Washington were given an instrument called the Life Events Inventory on which they reported about the likelihood of various childhood events. Based on their scores on one critical item (i.e. a score lower than 4), 72 subjects were selected and asked to participate in the next phase of the study. Of the selected 72 subjects, half were randomly assigned to the experimental condition (Dream), where subjects received the suggestive information about the content of their dream, and half were assigned to the control condition (Control), where subjects did not receive any suggestive information about the content of their dreams. Of the 36 Dream subjects, only 27 completed all three phases of the experiment; all 36 Control subjects completed the experiment. The mean age of the final sample of 63 subjects was 20 years. Of the 63, 54 were females and 9 were males.

\section{Materials}

A 20-item Life Events Inventory (LEI) was administered twice during this study. The inventory asked subjects to consider how confident they were that each event had or had not happened to them before the age of 3. Subjects responded by crossing the appropriate numeral on an 8-point rating scale, where 1 indicated 'definitely did not happen' and 8 indicated 'definitely did happen'. Of the 20 items, some referred to positive events (e.g. Adopted a lost animal), some to negative events (e.g. Hand caught in a mousetrap), and some to relatively neutral events (e.g. Found some lost keys). Among those 20 items, one item was selected to be critical, 'Was harassed by a bully', and it was randomly assigned the eleventh position in the LEI. The selected item had three characteristics: (1) it referred to a potentially distressing event; (2) we could expect to find sufficient individuals who would rate the events as being unlikely to have occurred to them before the age of three; (3) it had the appearance of being a profound and meaningful, as opposed to trivial, experience for a subject had it happened. We also decided to use a second item as a critical one in case insufficient numbers of subjects gave initially low scores to the 'Bully' event. We chose to use the item 'Was lost in a public place for more than one hour' to guide subjects to a highly 
specific belief, not the simple generic idea of getting lost, however briefly. Also, a variation of the getting lost belief had already been successfully used in our prior research (Mazzoni et al., 1997). This 'lost' item was the critical one for five of the final Dream Interpretation subjects.

All subjects filled out a 'Dream Survey' which contained 12 questions on Dreams and 12 questions on Sleep. Most of the questions were simply filler items, such as 'How often do you remember your dreams?' However, five of the questions concerned the subject's views on dream interpretation, and we refer to these as the Beliefs About Dream Interpretation (BADI) items. The BADI questions were placed midway in the Dream Survey and subjects responded to these by circling a number between 1 indicating 'never' and 5 indicating 'always': (a) Do you think your dreams are pictures of reality? (b1) Do you think that dreams have meaning? (b2) Do you think that this meaning is accessible through dream interpretation? (c) Do you believe that dream interpretation is valuable? (d) Do you think that you can discover yourself through analysis of your dreams?

Dream subjects and a subset of the Control subjects were asked to complete a Dissociative Experience Scale (DES). This 28 -item instrument taps into the extent to which a person has lapses in memory and attention. Subjects were asked to rate how often each of the 28 events happens to them. Items took the form 'Some people have the experience of driving or riding in a car or bus or subway and suddenly realizing that they don't remember what has happened during all or part of the trip. Circle a number to show what percentage of the time this happens to you.' The options ranged from $0 \%$ to $100 \%$ in steps of 10 .

Subjects also completed a series of filler tasks (mental rotation, mental manipulation of images, creation of mental images, etc.). A subset of the subjects also gave some memory ratings for the LEI events, but these items were confusing and ambiguous and were not analysed further.

\section{Procedure}

Figure 1 presents an overview of the study, indicating how many subjects completed the various parts of the study. Most of the Control subjects participated in two sessions. In session 1, they completed the 20-item LEI and the 24-item Dream Survey (which included the five BADI items) administered in a mass testing setting. They returned after 3-4 weeks for their second session administered in a laboratory room in the Psychology Building. The Dream subjects also had the same two sessions. In between the two sessions, however, they would partake in what they thought was a completely different experiment on dream interpretation. The cover story for sessions 1 and 2 told subjects that the study concerned the frequency of rare and common events that happened during early childhood (Early Childhood Study), and that the study goal was the validation of an instrument to measure these experiences. The cover story for the Dream interpretation session told subjects that this was a new study about the relationship between sleep, dreams, and cognition (Dream and Cognition Study).

For the Dream subjects, the dream interpretation was held approximately 1.5 to 2 weeks after session 1. Subjects in the Dream group participated individually, completed the filler tasks and the DES, and subsequently had their dreams interpreted by what we call here the 'clinical psychologist'. We had two 'clinical psychologists' 


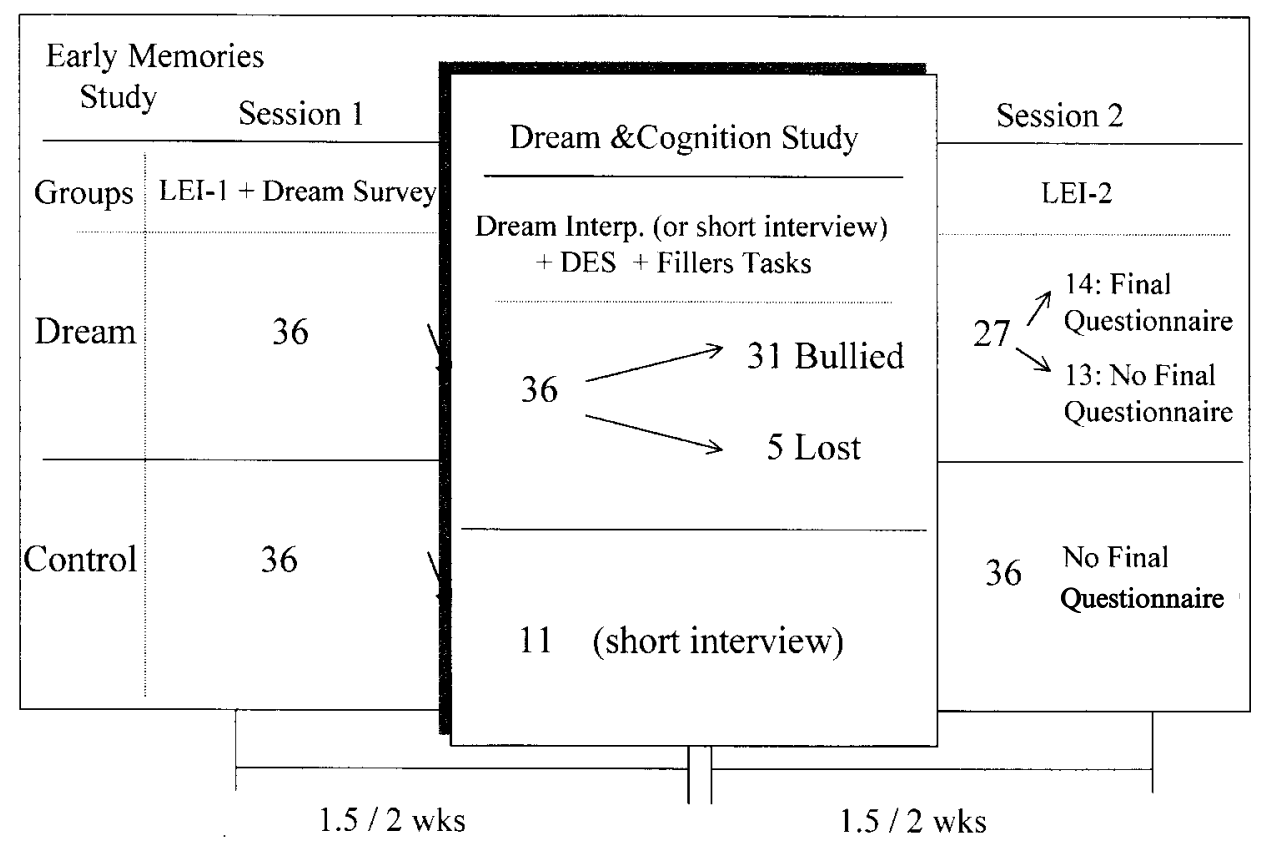

Figure 1. Overview of the procedure

interpreting dreams. One (also the first author) was a trained clinical psychologist with 16 years of experience in a private psychotherapy practice. The other individual (the third author) was trained by the clinical psychologist to use identical dreaminterpretation procedures. Both of the experimenters who conducted the Dream sessions introduced themselves as individuals with vast experience in Dream interpretation.

Shortly before the dream interpretation session, Dream subjects received a phone call from an experimenter asking for their participation in a dream and cognition study. They were asked to bring in two dreams, that could be either a recurrent dream, a recent dream, the only vivid dream of their life, etc. (no constrains were put on the type of dream). In the dream session, the 'clinical psychologist' welcomed the subject, explained that the purpose of the study was to collect meaningful dreams and relate those dreams to cognitive characteristics such as the ease of manipulating visual images.

Since dream interpretation is the key aspect of the experiment, we expand on this. A key feature of the dream manipulation was to suggest to subjects that the dream was the overt manifestation of repressed memories of events that happened before the age of 3. Specifically, the dream interpretation was designed to suggest to the subject that the dream was indicating a difficult childhood experience such as being bullied (for 22 of the 27 Dream subjects), or getting lost in a public place (for 5 of the Dream subjects), i.e. the critical item. No matter what the content of the subject's dream, all subjects received the same suggestion that the critical experiences appears to have happened to them before the age of 3 .

The dream interpretation process began by asking subjects for their interpretation of the dream, and for their comments on the dream. Then the 'clinical psychologist' 
offered specific comments. These were framed in terms of a clinical interview (i.e. the psychologist followed a predefined script, but was free to make some modifications depending upon the responses of the subject). Early on, the clinician claimed to possess considerable experience in dream interpretation, and explained that dreams are meaningful and symbolic expressions of human concerns.

To appreciate what the clinician did with the specific dream material, it is helpful to use a concrete example. One subject came in with two dream reports. One was about her house being on fire, and with her mother trying to help but finding only dangerous men around. The other dream was about her sister getting sick and she getting lost and hurt in the woods trying to get help for the sister. When asked by the clinician for the subject's thoughts about the dreams, the subject commented that in both situations she was trying to be of help in some life-threatening situations. The clinical psychologist then discussed the dreams and explicitly mentioned some key elements (house on fire; sister's sickness; dangerous men; being hurt in the woods), stressing how all referred to anxiety-arousing and life-threatening situations. The interpretation went on to mention that in both dreams the subject's attempt to get help and to find a positive resolution for the situation was unsuccessful. At this point the clinician tried to induce the subject to agree with this suggestion, encouraging the subject to provide any episodes in the subject's life that would confirm the clinician's interpretation. The psychologist then moved towards a 'global interpretation', and proposed some generic considerations about the subject's personality. For example, she suggested to the subject that in her vast experience with dream interpretation, a dream like this usually means that 'the world is dangerous' and that fear and inadequacy are among the subject's strongest feelings and emotions. She again encouraged the subject to confirm this interpretation.

She then suggested to the subject that the dream content, and the feelings about that dream, were probably due to some past experience that the subject might not even remember. The specifics that the subject mentioned are commonly due, it was suggested, to having had certain experiences before age 3, like being bullied by an older child. The subject was then told that although memory for experiences before age 3 is very poor, children still experience strong emotions at that age that, even if not verbalized in an adult manner, can be sufficiently strong to 'shape' reactions during adulthood.

Finally, the clinician asked whether the critical event (e.g. being bullied by an older child) happened to the subject before the age of 3. Most subjects at this point claimed not to remember, and when this happened, the clinician explained how childhood experiences are often buried in the unconscious, but do get revealed in dreams.

From this example, it is easy to see some of the general steps that the clinician followed during dream interpretation:

- She commented on specific items in the dream, and tried to relate those items to possible feelings that the subject might have. In the example, the specific items of fire or sickness, and the danger while trying to get help, indicate high anxiety.

- She tried to induce the subject to agree with and expand upon her interpretation.

- She provided a global interpretation of the dream meaning. In the example, the clinician suggested that possibly the subject considered the world a potentially 'dangerous' place, and felt inadequate to cope with such risky situations. 
- She suggested the possibility that specific events of childhood are commonly associated with dream reports such as the one provided by the subject. In the example, the specific events were having faced a bully (bigger, aggressive, physically harmful person).

- She explicitly suggested that such event had happened to the subject, and asked for the subject's agreement.

- When the subject did not recall such an event, she explained to the subject how unpleasant childhood experiences can be buried, remain unremembered, but are often revealed in dreams.

The entire dream session lasted approximately 30 minutes. At the end of the dream session, the subject was invited to think over the proposed event, and to feel free to come again and discuss further points with the 'clinician'. None of the subjects returned for this proffered discussion.

As for Control subjects, 25 of them participated in two administrations of the LEI, separated by $3-4$ weeks, with no intervening session. We had intended to use a second Control group that would not only participate in the two administrations of the LEI, but would also complete a brief intermediate session so as to be treated in a way that was more similar to the Dream subjects. This brief intermediate session, occurring 10-15 days after session 1, included the completion of the filler tasks, the DES, and a 5 -minute session with a 'clinician' discussing dreams in general, without mentioning any specific dreams of the subject or their interpretation. Because of scheduling difficulties we were able to obtain data from only 11 Control subjects with this procedure. These two subsets of Controls, totalling 36 subjects, comprised the total Control group.

In the final session, Dream and Control subjects were contacted by the initial experimenter who had previously administered the LEI (hereafter LEI-1), and arranged for the subjects to return for a second administration of the LEI (hereafter LEI-2). Just to be clear, about 1.5 to 2 weeks passed between LEI-1 and Dream Interpretation, and about another 1.5 to 2 weeks between Dream Interpretation and LEI-2. For all control subjects the LEI administrations were separated by the same amount of time.

After filling out the rating scales for the second LEI, subjects were asked to provide specific memory reports for several events from their second LEI. Again, these were supposed to be memory reports from before the age of 3 . The instructions were as follows: 'Earlier you filled out an LEI. I am studying autobiographical memories of early childhood, and am examining the completeness and quality of such memories. Today, we are checking the reliability of the LEI. Would you describe to me the memory you have for these five events? Try to remember as much as you can, but please try to spend only three minutes on each question.' The experimenter then read an item number, and the item description: for example, 'Item 14, won a stuffed animal at a carnival game'. Subjects were always asked to write about five items, and the critical item was in position number three. 'Bully subjects' for example, were asked to write about 'item 11, was harassed by a bully'. 'Lost subjects' were asked to write about 'item 15, was lost in a public place for more than an hour'. Three of the other four items for which memory reports were solicited were randomly selected to be included on the list of five. The last of the non-critical items included in the set of five was the 'lost' items (for bully subjects) and the 'bully' item (for lost subjects). Subjects 
were explicitly instructed to tell us if they had no memories for the events being queried, but to provide details in writing if they did have a memory.

Next, subjects were thoroughly debriefed about the study. At this time a subset of the Dream subjects $(n=14)$ completed a final questionnaire concerning their current beliefs about their recovered memory and their understanding of the experiment. Subjects responded by placing an $\mathrm{X}$ to indicate their answers, or by circling a number ranging from 1 (no effect at all) to 7 (large effect).

\section{RESULTS}

To reiterate, the main aim of the experiment was to assess whether a 30-minute dream interpretation, where the clinician suggests that the dream content is the sign of a buried memory about early childhood, increased subjects' confidence that a certain event occurred in childhood. Once we observed that confidence was increased, we explored whether subjects also generated a concrete memory report.

Recall that subjects were selected because they indicated on LEI-1 that the critical event had not happened in their early childhood (ratings between 1 and 3 on an 8-point scale). To determine whether dream interpretation caused these subjects to become more confident that the critical events had occurred, we examined whether subjects moved up or down in terms of their LEI score. In other words, we calculated, for the critical item (item 15 (Lost) for 5 subjects and item 11 (Bullied) for the remaining 22 subjects), the percentage of subjects whose responses increased, decreased, or did not change from the first to the second administration of the LEI. We first assessed whether the two Dream session experimenters produced different results in subjects who underwent Dream interpretation $(N=27)$. For this purpose, subjects who increased their score in the critical item in LEI-2 compared to LEI-1 were assigned a score of 3 , those who gave the same rating were assigned a score of 2 and subjects who decreased their score were assigned a score of 1 . On these scores we computed a MannWhitney test, comparing the group that had the dream interpreted by the clinical psychologist and the group that had the dream interpreted by the trained nonclinician. The Mann-Whitney $U$ was not significant, $U=400, z=0.30, p=0.77$. The data from all subjects were therefore collapsed across the two clinicians for the subsequent analyses.

\section{Analysis of 'bully' subjects}

First we examined only the data on the 'bully' critical item. (For this subset of data, the two clinicians' data were equivalent, $U=302, z=0.87, p=0.38$.) The collapsed data are shown in Figure 2. We predicted that Dream subjects would be more likely to move in the direction of becoming more confident that the bully event had happened.

First, examine what happens without dream interpretation, that is, with Control subjects. (The two types of Control subjects were not significantly different from each other and were thus combined into a single Control group.) The 36 Control subjects, who did not have any dream interpretation between the two administrations of the LEI, rarely increased their confidence that the critical event happened. Only $11 \%$ did so, whereas $31 \%$ reported a lower confidence score, and $58 \%$ reported the same score. Not so after Dream interpretation. The 22 Dream subjects produced scores that 


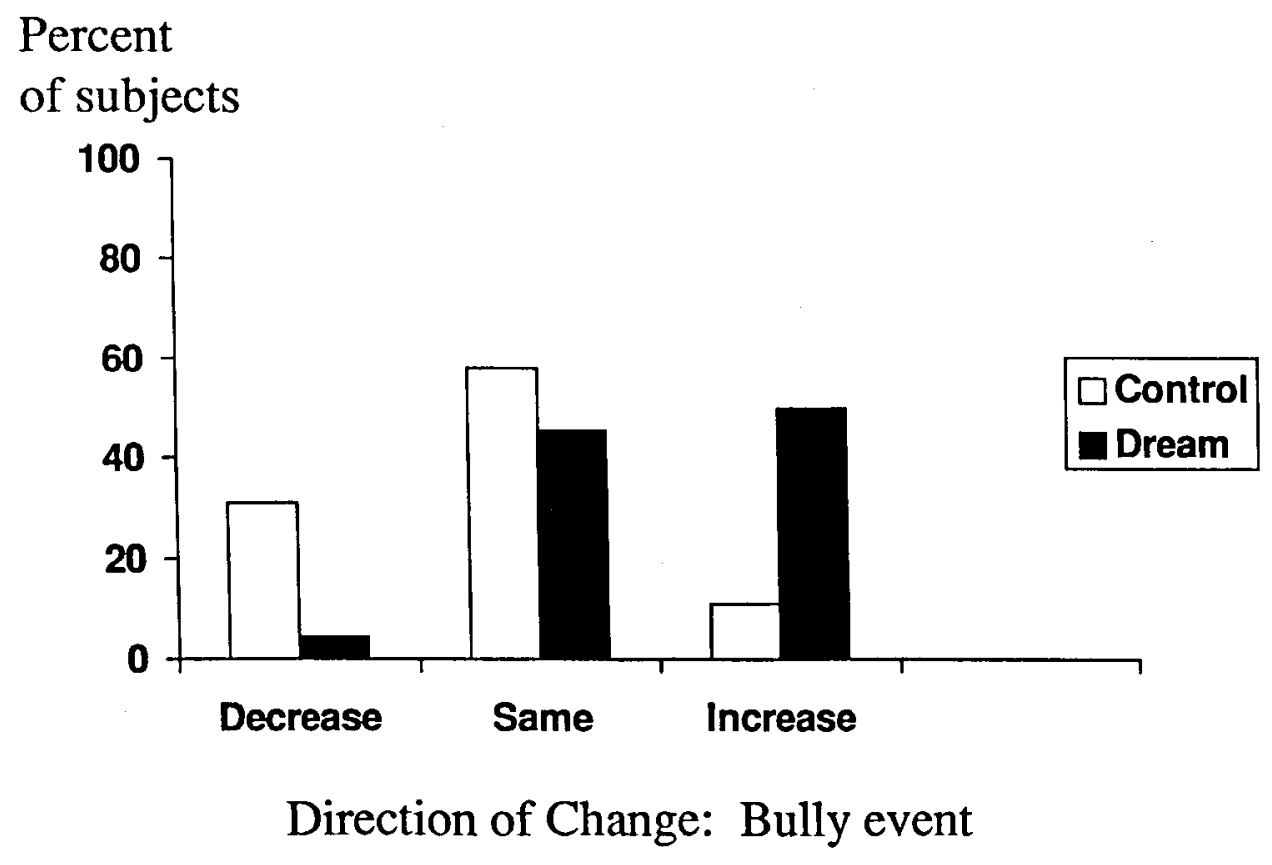

Figure 2. Percentage of subjects who decreased, who stayed the same, and who increased their scores for the critical item on the Life Events Inventory

were more likely to increase $(50 \%)$ and they rarely decreased on the second LEI $(4.5 \%)$, while nearly half stayed the same $(45.5 \%)$.

To analyse these data statistically, we assigned numerical scores to each subject depending on whether they increased (score of 3), remained the same (score of 2) or decreased (score of 1). We then performed Mann-Whitney $U$ tests, comparing Dream to Control conditions. The two groups differed significantly, Mann-Whitney $U=197.5, z=3.51, p<0.001$.

We also analysed the data in a different way, one that takes into account not only whether subjects increased or decreased in confidence, but also the degree of movement. We calculated for each subject the numerical difference between the scores assigned to each item in LEI-2 and those assigned to the same items in LEI-1. As we did for the previous data (on the percentage of subjects that increased, decreased or gave the same score), as well as for difference scores we first assessed whether the two clinical psychologists produced different results. We computed a $t$-test for independent samples on difference scores. The $t$-test was not significant, $t(56)=1.12, p=0.27$. Therefore data on difference scores for the critical item were collapsed across the two experimenters.

We found that for Control subjects the mean change in LEI scores was relatively small, and negative $(-0.50)$, meaning that they were slightly less likely to believe that the critical event happened on LEI-2. For the Dream subjects the mean change was larger and in the predicted positive direction (1.18), meaning that they were more likely to believe that the critical event happened on LEI-2. To analyse these data statistically, we computed a Student's $t$-test for independent samples on the change scores. The difference was highly significant $t(56)=3.44, p<0.001$. 
Thus, the two methods of analysis, one that involved percentage of subjects who shifted and one that involved measures of mean shift, produced very similar results. The findings suggest that the Dream manipulation caused subjects to become more certain that they had been bullied in childhood before the age of 3 .

\section{Analysis of all subjects}

Next we present, for completeness, the collapsed data for all subjects, those who had the bully item and those five who had the lost item as their critical item. Thus these data are based on 27 subjects who went through dream interpretation. Again, we first examine what happens without dream interpretation. To create a meaningful Control group, we needed to not simply use the 'bully' data from the control subjects. Since we had five 'lost' subjects in the Dream interpretation group, we randomly selected seven subjects from the 36 Controls, and used their LEI scores on the 'lost' item. These seven had to have had low initial 'lost' LEI scores to be included. (The reason that seven were selected was to keep the proportion of 'lost' subjects in the Control condition equivalent to the proportion of 'lost' subjects in the Dream condition. In other words, the lost subjects made up about $19 \%$ of the sample.) We combined these with the LEI scores on the 'bully' item produced by the remaining 31 Control subjects. These procedures constituted our Control data for the 'critical event' for subsequent analyses.

These Control subjects were more likely to report a lower $(28 \%)$ or the same score $(58 \%)$ than a higher one $(14 \%)$ on the second LEI. In fact the scores were twice as likely to decrease than increase. Not so after Dream interpretation. The scores were more likely to increase $(52 \%)$, and they rarely decreased $(7 \%)$ on the second LEI, while $41 \%$ produced the same score.

To analyse these data statistically, we assigned numerical scores to each subject depending on whether they increased (score of 3), remained the same (score of 2) or decreased (score of 1). We then performed Mann-Whitney $U$ tests, comparing Dream to Control conditions. The two groups differed significantly, Mann-Whitney $U=261, z=3.43, p<0.001$.

We also analysed the data in a different way, one that takes into account the degree of movement. We calculated for each subject the numerical difference between the LEI-2 and the LEI-1 scores for all 27 subjects who went through dream interpretation. Also, for this larger set, we assessed whether the two 'clinical psychologists' produced different results. The $t$-test for independent samples was not significant, $t(61)=0.71$, $p=0.48$. Therefore data on difference scores in the critical item were collapsed across the two clinicians.

We found that for the 36 Control subjects, the mean change in LEI scores was relatively small, and negative $(-0.47)$. For the Dream subjects the mean change was larger and in the predicted positive direction (1.15). To analyse these data statistically, we computed a Student's $t$-test for independent samples on the change scores. The difference was highly significant $t(61)=3.49, p=0.001$.

The two methods of analysis, one on the percentage of subjects who shifted and one on mean degree of shift, produced very similar results. Thus, based on the total sample, Dream manipulation caused subjects to become more certain that they had had a specific rather negative experience in their early childhood.

A question arises as to whether the shifts are localized only to the specific experiences mentioned by the clinician, or whether the clinician's intervention caused a 

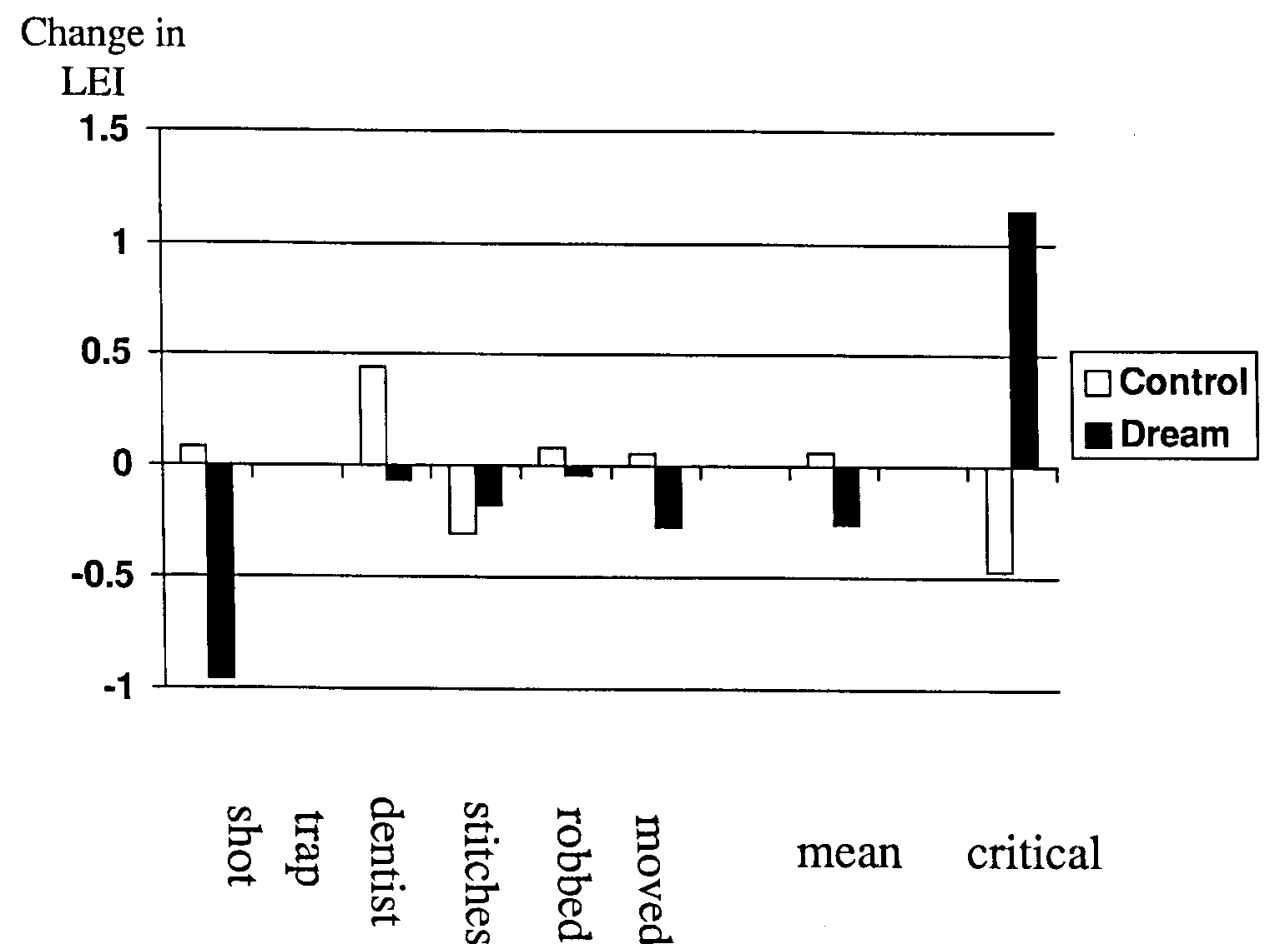

Figure 3. Mean change in LEI scores for the negative filler items. Towards the right is the mean change collapsed across all six negative filler items and, finally, the mean change score for the critical item

general negative feeling, creating in subjects the belief that they were more likely to have experienced a vast array of negative events in their early lives. We assessed this possibility by examining the Dream versus Control differences on the negative filler items on the total sample. If the Dream manipulation produced general negativity, this might be represented in greater increased confidence on negative filler items, compared to Control responses on those negative filler items. Figure 3 shows the list of negative filler items, and the mean Dream and mean Control change scores for those items. In general, some of the negative filler items increased slightly in negativity after Dream interpretation, some decreased, but no systematic pattern emerged. In other words, it was not the case that the Dream session caused subjects to be more confident that negative experiences in general had happened to them in early childhood.

So, for example, filler item 2 was 'had a shot at the doctors', and change in LEI score for this item is represented by the first two vertical bars. Whereas the mean change score was 0.08 for the control subjects, the change was -0.96 for the Dream subjects, indicating that Dream subjects tended to decrease confidence that this event happened. In fact, this difference was significant, $t(61)=2.51, p=0.015$, but in the direction of a decrease, not an increase, in the belief that this event had occurred. For the remaining negative items difference scores between Dream and Control subjects were never significantly different. (The smallest $p$-value for a difference was 0.28 .) For item 7 'cried at the dentist', item 17 'house robbed', and item 5 'best friend moved' Dream subjects slightly decreased their confidence $(-0.07 ;-0.04$ and -0.26$)$ and Control subjects 
slightly increased $(0.44,0.08$ and 0.05$)$. For item 3 , 'had my hand caught in a mousetrap', the difference score was 0 for both groups. Item 9, 'fell off a tricycle and got stitches in my leg', produced change in LEI that was negative for both Dream and Control subjects. The mean change in LEI collapsed across all six negative filler events can also be seen toward the right portion of Figure 3. Both subjects in the Control and in the Dream conditions changed only slightly ( 0.05 and -0.25 respectively). To get a feel for the small magnitude of these changes, the mean changes for Control and Dream subjects on the critical items are displayed for comparison purposes in the two rightmost bars in Figure 3.

Another way to ascertain whether the Dream manipulation simply produced a general negativity is to examine the changes that occurred on the positive filler items. A general negativity might lead Dream subjects to believe that they were less likely to have experienced positive events in the past. We assessed this possibility by examining the Dream versus Control differences on the positive filler items. If the Dream manipulation produced general negativity, this might be represented in less confidence about the positive filler items, compared to Control responses on those positive items. Figure 4 shows the list of positive filler items, and the mean Dream and mean Control change scores for those items. In general some of the positive filler items increased, some decreased, but no systematic pattern emerged. In other words, it was not the case that the Dream session caused subjects to be less confident that positive experiences in general had happened to them in early childhood.

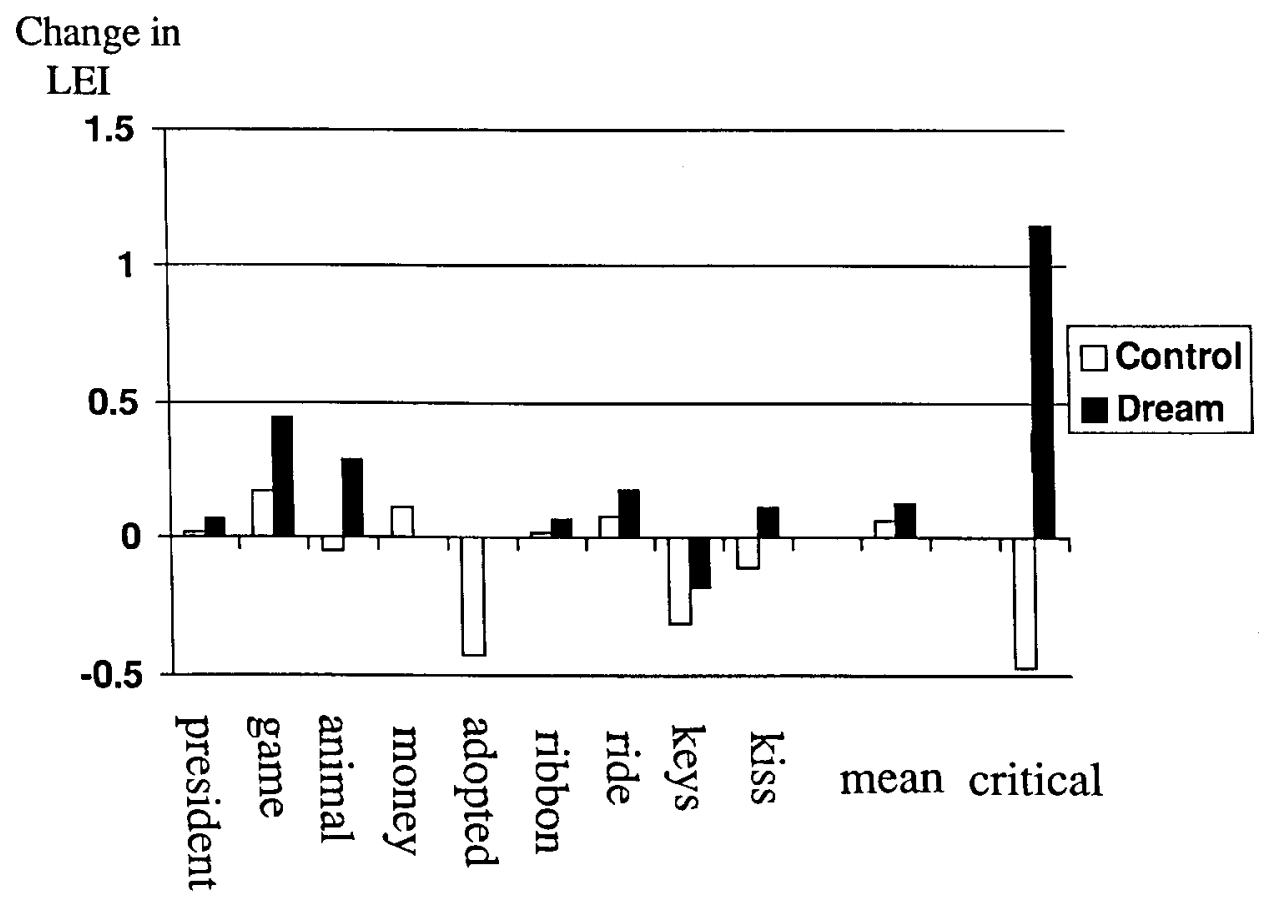

Figure 4. Mean change in LEI scores for the positive filler items. Towards the right is the mean change collapsed across all nine positive filler items and, finally, the mean change score for the critical item 
So, for example, filler item 1 was 'shook hands with the president' labelled 'president' in Figure 4. The mean change score on this item was 0.00 for Control subjects and .07 for Dream subjects. Item 14 was 'won a stuffed animal' labelled 'animal' in Figure 4. In this case Dream subjects showed a greater tendency to increase their score $(0.44)$ than Control subjects $(0.17)$. Of the nine positive filler items, seven showed that the Dream subjects were more likely to move in a positive direction (thinking the positive event was more likely to happen) compared to Controls. This is exactly opposite to what would be expected by the hypothesis that the Dream manipulation had produced general negativity. Separate $t$-tests on the nine items showed that in only one case ('president') were Dream subjects significantly different from Controls, but in a direction opposite to that predicted by general negativity, $t(61)=1.67, p=0.04$.

The mean change in LEI collapsed across all nine positive filler events can be seen toward the right portion of Figure 4. Both subjects in the Dream condition and subjects in the Control condition changed only slightly ( 0.14 and -0.03 , respectively). To get a feel for the small magnitude of these changes, the mean changes for Control and Dream subjects on the critical items are once again displayed for comparison purposes. In sum, Dream subjects did not show lower confidence that positive events had happened to them in the past than Control subjects.*

\section{Additional analyses: memory reports}

The previous analyses reveal that the Dream interpretation increases confidence in the critical event, relative to controls. But how frequently do subjects go on to produce a memory report when subsequently probed for details? To determine this, we divided the Dream subjects into two groups, those who increased in LEI score for the critical item and those who did not. We further subdivided the subjects into those who produced a memory report and those who did not. The 'no memory report' subjects actually wrote 'no memory', 'cannot remember this really happening', or something similar. The 'memory report' subjects gave some indication of having a memory. Figure 5 displays the results of these calculations.

Notice that for those subjects whose LEI scores increase, over half $(57 \%)$ went on to produce a memory report. In contrast, for those subjects whose LEI scores did not increase, only $30 \%$ produced a memory report, and the vast majority $(70 \%)$ did not. Thus, there does appear to be something special about the group of subjects who increased in LEI, after the Dream session, in that so many more of them came up with a memory report. By the way, one might be interested in the behaviour of subjects who increased in LEI after no Dream session (Controls), in terms of their propensity to produce a memory report. Only four Control subjects increased in LEI

\footnotetext{
*We computed the same analyses on the 22 'bully' Dream subjects, and obtained very similar results for both the negative and the positive set of items. For the item 'shot at the doctors' the difference score was 0.73 ; for 'hand in mousetrap' it was 0.23 ; for 'dentist' it was 0.14 ; for 'stitches in my leg' it was -0.23 ; for 'house robbed' it was -0.04 , and for 'best friend moved' it was -0.14 . The overall change score was 0.12 . We also report the difference scores for the positive items. For the item 'shook hands' the difference score was 0.04 ; for 'sport game' it was 0.27 ; for 'won a stuffed animal' it was 0.77 ; for 'found money' it was 0.23 ; for 'adopted animal' it was 0.04 ; for 'won blue ribbon' it was 0.29 ; for 'first airplane ride' it was 0.41 ; for 'found keys' it was 0.32 ; for 'kissed' it was 0.33 . The mean difference scores was 0.30 . All difference scores in the Dream group were more likely to move in a positive direction. This is opposite to what would be expected if Dream manipulation had produced general negativity.
} 


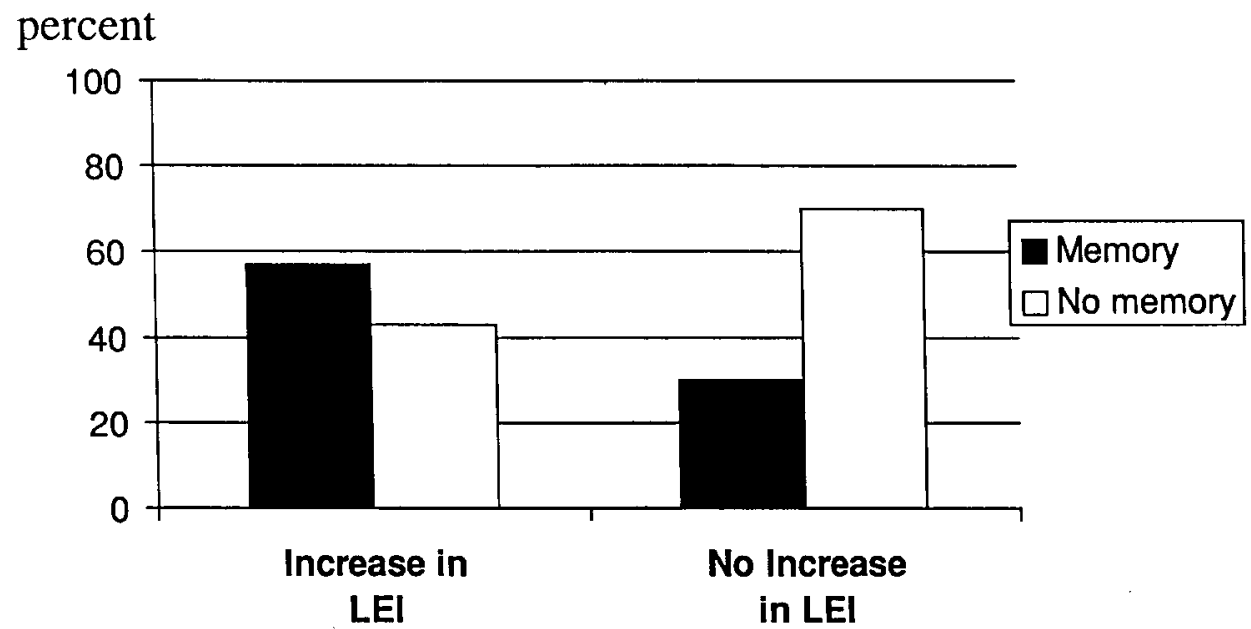

Figure 5. Percentage of Dream subjects who do and do not report a memory for the critical event. Dream subjects who increased in LEI were far more likely to report a memory than those who did not increase in LEI

score, and only a single one of these produced a memory report. Thus it was very rare for the Control subjects to both increase their LEI and also to produce a memory report $(1 / 36=2.7 \%$ of the Control sample).

We were most interested in the eight Dream subjects who increased their LEI score on the critical item and produced a memory. Of the total sample of 27 Dream subjects, eight $(29 \%)$ fell into this intersection. Five of these reported a 'bully' memory and three reported a 'lost' memory. Their data are shown in Table 1.

Table 1. Memory reports of the eight Dream subjects whose LEI scores increased

\begin{tabular}{|c|c|c|c|}
\hline \multirow[b]{2}{*}{ Subject } & \multicolumn{2}{|c|}{ LEI change } & \multirow[b]{2}{*}{ Memory reports } \\
\hline & LEI-1 & LEI-2 & \\
\hline \multicolumn{4}{|l|}{ 'lost' } \\
\hline NL & 1 & 5 & $\begin{array}{l}\text { 'My parents and I went shopping at Bellevue Square, and I ran } \\
\text { off when they went to look at some clothes for me. I had to wait } \\
\text { in the security office until my parents came.' }\end{array}$ \\
\hline NF & 1 & 3 & $\begin{array}{l}\text { 'I remembered standing by myself at the zoo looking for my } \\
\text { parents. I felt lost, but found out my parents were hiding \& } \\
\text { taking pictures. I wasn't crying, just being observant.' }\end{array}$ \\
\hline EG & 1 & 3 & $\begin{array}{l}\text { 'I was walking beside my mother in a public place, so there is a } \\
\text { chance I got lost in a public place.' }\end{array}$ \\
\hline \multicolumn{4}{|l|}{ 'bully' } \\
\hline MG & 1 & 8 & $\begin{array}{l}\text { 'I remember that a little boy would kick my leg under the table } \\
\text { when I was in preschool.' }\end{array}$ \\
\hline MW & 3 & 8 & $\begin{array}{l}\text { '... at camp a friend (girl) \& I were harassed by a group of older } \\
\text { boys - made fun of us \& sometimes nice.' }\end{array}$ \\
\hline $\mathrm{NE}$ & 1 & 5 & $\begin{array}{l}\text { 'I recall being harassed by An, but I never though of him as a } \\
\text { bully. He only did it once, and later we became friends. I don't } \\
\text { remember what it was about, but it occurred (sic) in first grade in } \\
\text { Mrs. Martin's class.' }\end{array}$ \\
\hline ES & 2 & 4 & 'When I was in 3rd grade I had another girl making fun of me.' \\
\hline PH & 2 & 4 & 'Probably when I was playing outside the house.' \\
\hline
\end{tabular}


The first two subjects, NL and NF, appeared to produce very concrete specific 'getting lost' memories, one about getting lost in a particular shopping mall, and another about getting lost at the zoo. Their reports included sensory details (e.g. Who was there? How did they feel?). Were these two subjects simply trying to provide some details to please the experimenter? Interestingly, one of these two, NF, also wrote 'no memory' for all four of the non-critical items which included such common experiences as 'had a shot at the doctors' and 'your best friend moved' Thus she showed that she was perfectly willing to refrain from providing memories when she did not have them. The third 'lost' subject, EG, appears not to have a concrete memory but to be making a judgement of the plausibility of the experience, since after mentioning walking with her mother in a public place infers that '... there is a chance I got lost once in a public place'.

The next two subjects, NMG and MW, produced 'bully' memory reports, one about being kicked on the leg by a boy and the other about being harassed by older boys while at camp. However, they seemed to involve multiple episodes, and it is unclear whether the subject was actually remembering a single individual concrete instance. In both cases, these individuals changed rather dramatically in their LEI scores, ending with scores of 8.

The next two subjects, NE and ES, produced 'bully' memory reports, one about being harassed by a friend in the first grade, at age 6 , and the other about being made fun of by a girl in the $3 \mathrm{rd}$ grade, when she was 8 . One of these reports clearly refers to a single episode that is remembered (NE), while the other appears to refer to multiple episodes. However, in both cases, the subjects have produced reports of events that occurred later than age 3. Both individuals increased their LEI from 1 to mid-range (4 and 5). Interestingly, for NE, who increased from 1 to 5 , three of the filler items began with LEI scores of 1 and did not change at all. And for each of these filler memory reports NE wrote 'never happened'.

The last subject, $\mathrm{PH}$, who wrote 'Probably when I was playing outside the house' appears not to have a concrete episodic memory for a single experience, or to even believe that there were multiple episodes, but instead appears to be making a judgment of the plausibility that the event happened, and to be guessing about where it might have happened.*

\section{Additional analyses: final questionnaire}

Of the special eight subjects in the Dream condition (who increased in LEI and also produced a memory report), only five were in the subset who were given the final questionnaire. Did they talk, read or think about the suggested event after the Dream session? Their responses indicated that they virtually never acknowledged talking or

\footnotetext{
*There are several additional subjects who came up with a memory report after the Dream session, and they increased their 'memory rating' score, although their LEI scores did not increase. One of these subjects, a bully subject, MS did not increase her LEI score (3 to 3) but did increase her memory rating ( 3 to 4 ). In her memory report she wrote 'have the memory, bullied by my brother, not very bad but enough to make me cry'. A second subject HW, a lost subject, did not increase her LEI score (3 to 3) but did increase her memory rating ( 3 to 4$)$. In her memory report, she wrote 'I was lost at a train station. I don't know how long I was by myself, but because it was a station I had never been before I was extremely frightened \& thought I'd never see my parents again.' The third subject SB, a lost subject, did not increase her LEI score (2 to 2) but did increase her memory rating (1 to 2). In her memory report, she wrote 'I'm sure I was @ one point or another. But I probably didn't consider myself lost, so I have no clear memory of this event occurring.'
} 
reading about their experience. All five acknowledged thinking about the event 'once or twice'. As for the reasons that they reported for changing their LEI scores, three said it was because of the dream session prompting them to remember a real event and two said it seemed more familiar. These three, who attributed their change to the dream session, all claimed that the event happened before age 3, and two of them said that they could provide corroboration, with the third being unsure. (Subjects EG and $\mathrm{PH}$ failed to respond to the age or corroboration questions.) None of these five subjects connected or suspected a link between the two experiments, although some of them indicated seeing some similarity. When asked if they thought the dream session affected their confidence in the critical event, three responded with a 1 or 2 indicating little or no effect, and two responded with a 5 on a 7 -point scale, indicating moderate belief that the dream session mattered.

\section{Additional analyses: individual differences}

Beliefs about dream interpretation (BADI)

We have shown that our dream interpretation session changed what people believe happened in their past. But not everybody in the Dream condition changed their confidence scores. We hypothesized that people might be more likely to change if they, a priori, attributed great importance to dreams and believed that dreams can reveal important information that is not accessible otherwise. To test this possibility we computed multiple correlations (Spearman) between difference scores and scores in the five Beliefs About Dream Interpretation (BADI) questions. None of the correlations was significant.

We then computed multiple correlations (Spearman) between the five BADI questions and the index about whether the subjects either increased, decreased or gave the same score in LEI-2 compared to LEI-1. In the Dream condition $(N=27)$, the correlation was significant for two items only: item (b2) which reflected their a priori belief that the meaning of dreams is accessible through dream interpretation $(r=0.40$, $p<0.05$ ) and item (d) which reflected their a priori belief that they can discover themselves through analysis of their dreams $(r=0.38, p<0.05)$. To be explicit about the direction of the correlations, the individuals who expressed high a priori belief in these aspects of dreams were more likely to increase their confidence that a critical event happened to them than subjects who did not hold these a priori beliefs about dreams. In the Control condition $(N=36)$, correlations were never significant.

\section{Dissociative Experiences Scale (DES)}

The 27 Dream subjects filled out the DES, rating how often each of 28 events happened to them $(0 \%$ of the time up to $100 \%$ of the time). A mean score was calculated for each subject, and then averaged across the sample. The mean DES score for the 27 Dream subjects was 22.7. We computed the Spearman correlation between the amount of change on the LEI and the score on the DES. This correlation was not significant in Dream subjects, $r=0.12, p>0.50$. Our next analysis was to divide the Dream subjects into two groups, those who increased their score on the LEI and those who did not. The mean DES for the increasing subjects was 23.4 , and for the non-increasing subjects it was 21.9, a difference that was not significant, $t(26)=0.29, p>0.2$. We next calculated 28 correlations, all between the amount of change on the LEI and the score for the individual DES items. All 28 correlations 
failed to reach statistical significance (all $p$ 's $>0.1$ ). The second largest correlation was with item 20 'Some people find that they sometimes sit staring off into space, thinking of nothing, and are not aware of the passage of time'. This produced a correlation of 0.28 . The largest correlation was for item 21 , 'Some people sometimes find that when they are alone they talk out loud to themselves', which produced a correlation of 0.31 . The seven subjects who both increased their LEI and also produced some memory report had a mean DES score 22.5 (which is indistinguishable from the total mean of 22.7). Thus it appears that the DES did not reliably predict who would change after Dream interpretation and who would not. The fact that the DES does correlate with suggestibility in other paradigms (e.g. Hyman and Billings, in press; Winograd et al., 1997) and not here might suggest that there are different mechanisms of manipulation being induced in these different paradigms. Perhaps the kind of suggestibility observed in the word intrusion studies is not the same as the kind of suggestibility that occurs in our dream paradigm.

For completeness, we report on the relationship between the DES and the degree of change in LEI for Control subjects. Eleven controls completed all questions required for this analysis, and the Spearman correlation was not significant, $r=0.48$, $p=0.15$. For completeness we also report that DES did not correlate with the five BADI questions.

\section{DISCUSSION}

We found that the 30-minute dream-interpretation session was sufficient to increase confidence that a certain critical event, either being bullied or lost before age 3, had occurred in the subject's childhood. The powerful impact of the dream session was readily apparent no matter whether the data were analysed in terms of proportion of subjects who increased their confidence, or of the mean change in confidence. Both the experienced clinician and the trained experimenter who had no prior clinical experience were able to achieve these results. The results appear not to be due to simple demand characteristics, because our data demonstrated that we successfully disguised the dream session and effectively convinced subjects that it was a completely unrelated study. So, impressively, subjects were reporting in a completely different setting, to a different experimenter, their belief that they had been bullied or lost. Finally, we found an individual difference variable that was associated with who was likely to change, namely the subjects' beliefs about dream interpretation. Those subjects who a priori had faith in dream interpretation and its ability to reveal important information were more likely to be influenced by our manipulation.

Some of our subjects not only changed their belief about their childhood, they also produced memory reports. We found that approximately $30 \%$ of Dream subjects increased their confidence and also produced a memory report. Only 3\% of Controls behaved this way. Some of the memory reports by Dream subjects were quite detailed. For example, one subject wrote 'My parents and I went shopping at Bellevue Square, and I ran off when they went to look at some clothes for me. I had to wait in the security office until my parents came.' Other subjects did not give detailed reports, even when pressed. For example, one subject wrote 'Probably when I was playing outside the house'. Thus, we have at least two ways in which autobiography was 
influenced by our manipulation, i.e. in the change of beliefs and in the production of new memories. Put another way, even if subjects did not come up with a memory report, but still demonstrated a significant shift in their confidence about this critical event (a changed belief but no new memory), we have in a sense still changed their autobiography. To see how this might have practical significance, imagine that some of our subjects prior to debriefing were to participate in a national telephone survey on people's recollection of school experiences. Imagine further that they are asked whether they had ever, in early childhood, been harassed by a bully. Some of our influenced research subjects would undoubtedly answer this question 'yes', signalling to the survey researcher that they had experienced this event in their past. This might artificially inflate the survey researcher's estimates of the prevalence of this experience, and lead to false results when the researcher attempts to relate the presence of the experience to other variables being measured.

\section{Memory reports}

We found eight individuals who both changed their belief (as measured by LEI changes) and also produced memory reports, and these instances provided evidence for the variety of ways people behave in this kind of situation. Some produced memory reports for experiences that were clearly after the age of 3. Some reported detailed memories that they claim occurred before age 3 . Some made inferences about what probably happened without providing any detailed episodes. These reveal the variety of ways in which memory is affected by our manipulation, and, in particular, the various ways in which people can come up with a 'new' memory that they hadn't apparently had when asked about the experience earlier.

Do these reflect true experiences or are they constructions in response to the suggestions from the clinician? We have no way of knowing for sure. In other research on planting false memories, investigators have received confirmation from family members that the suggested events did not happen (Loftus and Pickrell, 1995; Hyman et al., 1995) but we did not obtain such 'corroboration' in our research. The suggestion might have triggered some real memories of bullying events that occurred later in life. It also might have changed the interpretation of previously harmless episodes. Consider the example of the person reporting being kicked by a boy at her preschool. Until dream interpretation the subject might have considered it an innocuous episode but after dream interpretation the episode became an example of 'being bullied'.

Of course, some of our subjects insisted later that the concrete very detailed memory occurred before the age of 3 . If we assume that with childhood amnesia such prior-to-age-3 memories are not likely to be true, which is why we even used the 'age 3 ' criterion, then we may be able to assume that these are either constructions, events heard from somebody, or possibly experiences that occurred later in life and are mistakenly transported to the age 3 time frame. We also know that subjects initially denied the bully (or lost) experience, and now they have increased their belief, and come up with some sort of memory report. Even if our manipulation brought a true memory from beneath the curtain of consciousness up to centre stage, we have in some sense changed the person's autobiography. 


\section{Beliefs versus memories}

People showed they were influenced by the dream interpretation in a number of ways. Some of them changed their beliefs that they were bullied or lost at an early age without developing any specific concrete memory. Some changed their beliefs and also produced memory reports. We believe that the group that has only demonstrated a belief change might still have been influenced in critical ways. For example, it is likely that these individuals would have come up with a memory report had they been interviewed again. They also might spend more time than they would otherwise trying to search for a memory, particularly if they believe it will lead to some benefit if they find one. In their efforts to search, they might come up with a real memory, and it's an empirical question whether they would be better off 'knowing' about this memory or having it remain buried. And, in their efforts to search, they also run the risk of constructing a false memory, one that might be harmless, but also might not be.

On the surface it might appear as if those who produced a memory report actually had more radical change, than those who did not. However, the above analysis suggests that this may not be the case. And there are other reasons as well. Perhaps the Dream subjects with memory reports have found some relatively bland instance of being bullied or lost and were able to stop thinking about the whole subject since an 'answer' had been found. A specific instantiation of the belief had been 'recovered' or 'created' and no further search was needed. In contrast, the Dream subjects with no memory reports might be haunted (had they not been debriefed) by the thought that lurking beneath conscious awareness was some horrible experience waiting to be unearthed. This speculation suggests a prediction: If we had asked the Dream subjects who had no memory to report to tell us how unpleasant the unremembered experience probably was, they might rate it as more horrible than the remembered experiences for those who provided reports.

\section{Implications for therapy}

While generalizing to psychotherapy always needs to be done with caution, our results suggest that mental professionals have enormous power to influence the way people think about their past. If we, even the inexperienced 'clinical psychologist', were able, in a 30-minute session, to change people so dramatically, a change that lasted for two weeks, think about the power that resides in the hands of a therapist who sees a client weekly. This authority figure, who may offer interpretations of dreams, behaviour, thoughts, and other information provided by a vulnerable client, would likewise be expected to be able to influence that client in significant ways. Clients go to therapy with expectations that the therapist might be able to shed light on their experiences. If even our subjects, who were not clients and did not have such strong expectations, were ready to accept dream interpretations from a total stranger, to change their beliefs, and in some cases to produce what might be true or false memory reports, then extreme caution in offering interpretations would be in order. Recognizing the power of the type of suggestion we have studied here would be an important first step in resisting the temptation to engage in it, and would go a long way towards minimizing the use of risky practices that have so concerned recent commentators (Lindsay and Read, 1995; Poole et al., 1995). 


\section{ACKNOWLEDGEMENTS}

Part of this study was supported by a MURST grant to the first author. We thank Rhiannon Ellis, Judson Jones, Lyscha Ann Marcynyszyn, Sarah Morsbach for valuable assistance with collection and analysis of data.

\section{REFERENCES}

Bernstein, E. M. and Putnam, F. W. (1986). Development, reliability, and validity of dissociation scale. The Journal of Nervous and Mental Disease, 174, 727-735.

Hyman, I. and Billings, F. J. (in press). Individual differences and the creation of false childhood memories. Memory.

Hyman, I. E., Husband, T. J. and Billings, F. J. (1995). False memories of childhood experiences. Applied Cognitive Psychology, 9, 181-197.

Lindsay, D. S. and Read, J. D. (1995). 'Memory work' and recovered memories of childhood sexual abuse: Scientific evidence and public, professional, and personal issues. Psychology, Public Policy \& the Law, 1, 846-908.

Loftus, E. F. and Pickrell, J. (1995). The formation of false memories. Psychiatric Annals, 25, $720-724$.

Malinoski, P. T. and Lynn, S. J. (1996). The plasticity of very early memory reports. Unpublished manuscript, Ohio University.

Mazzoni, G. A., Lombardo, P., Malvagia, S. and Loftus, E. F. (1997). Dream interpretation and false beliefs. Unpublished manuscript, University of Florence \& University of Washington.

Poole, D. A., Lindsay, D. S., Menon, A. and Bull, R. (1995). Psychotherapy and the recovery of memories of childhood sexual abuse: U.S. and British practitioners' opinions, practices, and experiences. Journal of Consulting and Clinical Psychology, 63, 426-437.

Winograd, E., Peluso, J. and Glover, T. A. (1997, July). Individual differences and memory illusions created in the laboratory. Presented in the Society for Applied Research in Memory and Cognition, Toronto. 